\title{
The Mechanism Study of Common Flavonoids on Antiglioma Based on Network Pharmacology and Molecular Docking
}

\author{
Taiping Li, ${ }^{1}$ Yong Xiao, ${ }^{2}$ Zhen Wang $\mathbb{D},{ }^{2}$ Hong Xiao $\mathbb{D},{ }^{1}$ and Hongyi Liu $\mathbb{D}^{2}$ \\ ${ }^{1}$ Department of Neuro-Psychiatric Institute, The Affiliated Nanjing Brain Hospital of Nanjing Medical University, \\ Nanjing, China \\ ${ }^{2}$ Department of Neurosurgery, The Affiliated Nanjing Brain Hospital of Nanjing Medical University, Nanjing, China \\ Correspondence should be addressed to Hong Xiao; xhnkyy123@163.com and Hongyi Liu; njnkyylhy@163.com
}

Received 19 July 2021; Revised 30 August 2021; Accepted 28 December 2021; Published 31 January 2022

Academic Editor: Md. Areeful Haque

Copyright (c) 2022 Taiping Li et al. This is an open access article distributed under the Creative Commons Attribution License, which permits unrestricted use, distribution, and reproduction in any medium, provided the original work is properly cited.

\begin{abstract}
Background. Glioma is the most common primary intracranial tumor in adult patients. Among them, glioblastoma is a highly malignant one with a poor prognosis. Flavonoids are a class of phenolic compounds widely distributed in plants and have many biological functions, such as anti-inflammatory, antioxidant, antiaging, and anticancer. Nowadays, flavonoids have been applied to the therapy of glioma; however, the molecular mechanism underlying the therapeutic effects has not been fully elaborated. This study was carried out to explore the mechanism of selected active flavonoid compounds in treating glioma using network pharmacology and molecular docking approaches. Methods. Active ingredients and associated targets of flavonoids were acquired by using the Traditional Chinese Medicine Database and Analysis Platform (TCMSP) and Swiss TargetPrediction platform. Genes related to glioma were obtained from the GeneCards and DisGeNET databases. The intersection targets between flavonoid targets and glioma-related genes were used to construct protein-protein interaction (PPI) network via the STRING database, and the results were analyzed by Cytoscape software. Gene Ontology (GO) and Kyoto Encyclopedia of Genes and Genomes (KEGG) pathway enrichment analyses were performed and displayed by utilizing the Metascape portal and clusterProfiler R package. Molecular docking was carried out by iGEMDOCK and SwissDock, and the results were visually displayed by UCSF Chimera software. Results. Eighty-four active flavonoid compounds and 258 targets overlapped between flavonoid targets and gliomarelated genes were achieved. PPI network revealed potential therapeutic targets, such as AKT1, EGFR, VEGFA, MAPK3, and CASP3, based on their node degree. GO and KEGG analyses showed that core targets were mainly enriched in the PI3K-Akt signaling pathway. Molecular docking simulation indicated that potential glioma-related targets-MAPK1 and HSP90AA1 were bounded more firmly with epigallocatechin-3-gallate (EGCG) than with quercetin. Conclusions. The findings of this study indicated that selected active flavonoid compounds might play therapeutic roles in glioma mainly through the PI3K-Akt signaling pathway. Moreover, EGCG had the potential antiglioma activity by targeting MAPK1 and HSP90AA1.
\end{abstract}

\section{Introduction}

Glioma, originated from the neuroepithelium, accounts for $40 \% \sim 50 \%$ of brain tumors and is one of the most common primary intracranial tumors; among them, glioblastoma is a highly malignant one with poor clinical outcome [1]. Extensive studies have shown that flavonoids have a good therapeutic effect on glioma [2-4]; however, the underlying therapeutic molecular mechanisms of flavonoids on glioma are not stated clearly. Hence, a systematic exploration of the molecular mechanisms of flavonoids on glioma is critical. Network pharmacology is an emerging interdisciplinary discipline and has been applied to comprehensively analyze the functional mechanisms of traditional Chinese medicine [5]. It is also used to reveal the active ingredients of natural medicine treating glioma [6].

In this study, we tried to systematically identify the molecular mechanisms of flavonoids' antiglioma effects based on findings from network pharmacology and molecular docking. The flowchart of this study is shown in 
Figure 1. Our work portrays the ground view of antiglioma molecular mechanisms of flavonoids, which provided active compounds and therapy targets curing glioma.

\section{Materials and Methods}

2.1. Screening Active Ingredients and Predicting Related Targets. TCMSP (https://tcmsp-e.com/) is a unique systematic pharmacology platform for Chinese herbal medicines and is characterized by exploring relationships between compounds, targets, and diseases. TCMSP has been utilized to screen active flavonoid ingredients [7]. Flavonoids are categorized according to their molecular structures into flavones, flavonols, isoflavones, chalcones, flavones, and anthocyanidins [4], as shown in Table 1. Key parameters were taken into account, such as oral bioavailability (OB), drug-likeness (DL), and blood-brain barrier $(\mathrm{BBB}) . \mathrm{OB}$ is a measurement of the proportion of drugs entering the blood circulation. DL indicates the potential of a compound to be developed into a therapeutic drug with respect to its physical and chemical properties. BBB impedes drug distribution between blood and brain [8], which is a very important parameter in the treatment of glioma. In general, one compound is considered permeable across the BBB when its $\mathrm{BBB}$ permeability is larger than -0.30 . Active flavonoids were acquired by the following criteria: $\mathrm{OB} \geq 30 \%, \mathrm{DL} \geq 0.18$, and $\mathrm{BBB} \geq-0.3[9,10]$. Active compounds of flavonoids reported publicly in recent five years were also taken into analyses from $1^{\text {st }}$ January 2016 to $30^{\text {th }}$ May 2021. The active flavonoids were confirmed by PubChem (https://pubchem.ncbi.nlm.nih. gov/). The structures of compounds saved in SDF format were used to predict potential targets of active molecules by using Swiss TargetPrediction platform (http://www. swisstargetprediction.ch/), and the species were set as "Homo sapiens" and the probability was set larger than 0 . All the targets from UniProt database (https://www.uniprot.org/) were in standardized format.

2.2. Determination of Glioma-Related Targets and Common Targets with Active Components. Glioma-related targets were retrieved by using the GeneCards (https://www. genecards.org/) and DisGeNET (https://www.disgenet.org/ home/) databases with the keyword "glioma." Flavonoid targets and glioma-related genes were imported into Venny 2.1 (https://bioinfogp.cnb.csic.es/tools/venny/) to acquire common targets as the potential targets for further analyses.

2.3. Network Construction of Common Targets. A proteinprotein interaction (PPI) network was constructed using the STRING platform (version 11.5, https://string-db.org/), and "Homo sapiens" and Medium Confidence (0.4) were set. All the information derived from the STRING database was then imported into Cytoscape software (version 3.8.2) for visual display.

2.4. GO Function and KEGG Pathway Enrichment Analyses. GO is wildly used to study gene functions, including the biological process (BP), molecular function (MF), and cell component (CC) [11]. KEGG (http://www.kegg.jp/) is an integrated database of genomic, chemical, and system functional information and is extensively used to capture significantly enriched biological pathways [12]. The common targets of flavonoid-glioma were imported into the Metascape portal (http://metascape.org/gp/index.html/) for enrichment analysis. The top $20 \mathrm{GO}$ and KEGG pathway enrichment analysis results were visualized by clusterProfiler $\mathrm{R}$ package as the histogram or bubble graph with $p<0.01$ [13].

2.5. Molecular Docking. The most potential pathway and its related genes of flavonoids treating glioma were obtained. Then, these targets with the most promising ingredients were reconfirmed by using molecular docking. Crystal structures of related proteins were obtained from the RCSB Protein Data Bank (PDB, https://www.rcsb.org/) with high resolution and score, water was removed, while hydrogens were added by MGLTools software (version 1.5.6). Verified compounds in.mol2 format were acquired from the TCM@ Taiwan database (https://tcm.cmu.edu.tw/). Molecular docking was carried out using iGEMDOCK software (version 2.1) with default parameters. We selected the most potential proteins which had the lowest energy and determined their docking ligands using the SwissDock platform (http://www.swissdock.ch/docking/). The results were visually displayed by UCSF Chimera software (version 1.15).

\section{Results}

3.1. Active Ingredients of Flavonoid. According to the TCMSP database, 55 compounds were screened out with the thresholds of $\mathrm{OB} \geq 30 \%, \mathrm{DL} \geq 0.18$, and $\mathrm{BBB} \geq-0.3$ (Table 2). Twenty-nine compounds were ruled out due to the aforementioned screening conditions, but they have been reported to have antiglioma properties in previous studies $[2,3,14-65]$. We added them into our study to decipher the whole view of flavonoids' antiglioma molecular mechanism. As a result, a total of 84 active compounds were selected for further analysis. Quercetin, epigallocatechin-3-gallate, isoliquiritigenin, genistein, apigenin, kaempferol, and luteolin had 154, 140, 124, 97, 80, 63, and 57 targets, respectively. It revealed that these seven flavonoids probably played significant roles in curing glioma (Table 3 ).

3.2. Overlapping Common Targets of Flavonoid-Glioma. After exclusion of duplicated data, 5086 and 3097 gliomarelated targets were identified from GeneCards and DisGeNET databases, respectively, and 569 candidate targets of active flavonoids were integrated from Swiss TargetPrediction. Two hundred and fifty-eight intersection targets were obtained among these three gene sets and were used for further analysis (Figure 2).

3.3. Common Targets Network Construction. These 258 putative gene targets correlated with glioma were analyzed using the STRING database. A total of 258 nodes and 4407 


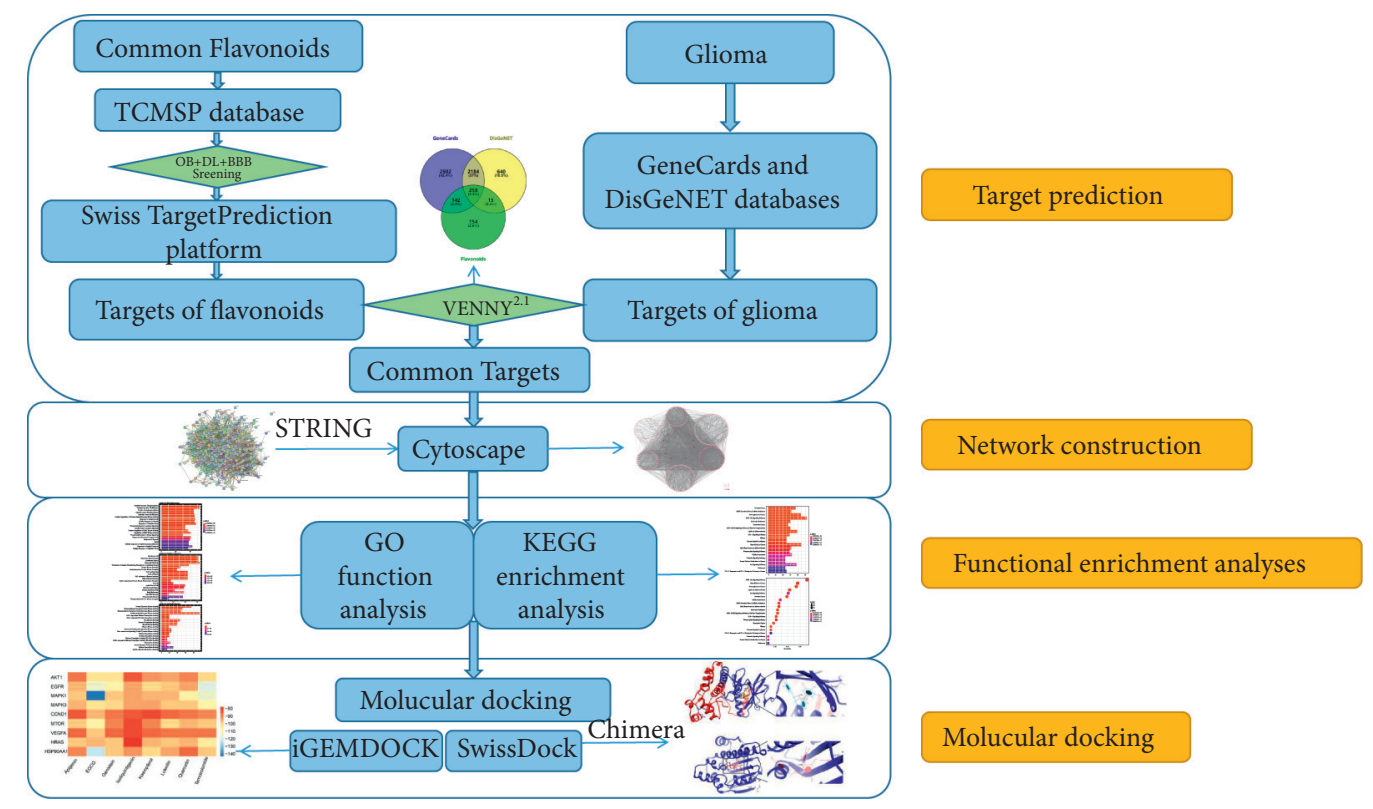

Figure 1: Study flowchart of the molecular mechanism of flavonoids in treating glioma.

TABLE 1: Main structure of flavonoids with their representative compounds.

Flavones

Flavonols

Isoflavones

Chalcones

Flavanones

Anthocyanidins<smiles>O=c1cc(-c2ccccc2)oc2ccccc12</smiles>

Apigenin, luteolin<smiles>O=c1c(O)c(-c2ccccc2)oc2ccccc12</smiles>

Quercetin, myricetin<smiles>O=c1c(-c2ccccc2)coc2ccccc12</smiles>

Genistein, daidzein

Isoliquiritigenin, corylifolinin

Naringenin, hesperidin

Cyanidin, pelargonidin 
TABLE 2: Basic information on the main active flavonoids.

\begin{tabular}{|c|c|c|c|c|c|c|}
\hline No. & Molecule ID & Molecule name & Related targets & $\mathrm{OB}(\%)$ & $\mathrm{BBB}$ & $\mathrm{DL}$ \\
\hline 1 & MOL000173 & Wogonin & 45 & 30.68 & 0.04 & 0.23 \\
\hline 2 & MOL003896 & 7-Methoxy-2-methyl isoflavone & 43 & 42.56 & 0.56 & 0.20 \\
\hline 3 & MOL000392 & Formononetin & 39 & 69.67 & 0.02 & 0.21 \\
\hline 4 & MOL002714 & Baicalein & 37 & 33.52 & -0.05 & 0.21 \\
\hline 5 & MOL005828 & Nobiletin & 35 & 61.67 & -0.08 & 0.52 \\
\hline 6 & MOL001876 & 6-Methoxyflavone & 34 & 34.56 & 0.49 & 0.18 \\
\hline 7 & MOL007879 & Tetramethoxyluteolin & 32 & 43.68 & 0.09 & 0.37 \\
\hline 8 & MOL000497 & Licochalcone A & 32 & 40.79 & -0.21 & 0.29 \\
\hline 9 & MOL004957 & $\mathrm{HMO}$ & 27 & 38.37 & 0.25 & 0.21 \\
\hline 10 & MOL004835 & Glypallichalcone & 27 & 61.60 & 0.23 & 0.19 \\
\hline 11 & MOL013277 & Isosinensetin & 27 & 51.15 & 0.03 & 0.44 \\
\hline 12 & MOL002928 & Oroxylin A & 26 & 41.37 & 0.13 & 0.23 \\
\hline 13 & MOL004828 & Glepidotin A & 26 & 44.72 & 0.06 & 0.35 \\
\hline 14 & MOL001689 & Acacetin & 26 & 34.97 & -0.05 & 0.24 \\
\hline 15 & MOL008206 & Moslosooflavone & 25 & 44.09 & 0.54 & 0.25 \\
\hline 16 & MOL004991 & 7-Acetoxy-2-methylisoflavone & 25 & 38.92 & 0.16 & 0.26 \\
\hline 17 & MOL008239 & Quercetin tetramethyl $\left(3^{\prime}, 4^{\prime}, 5,7\right)$ ether & 23 & 31.57 & 0.36 & 0.41 \\
\hline 18 & MOL005229 & Artemetin & 23 & 49.55 & -0.09 & 0.48 \\
\hline 19 & MOL008400 & Glycitein & 23 & 50.48 & -0.29 & 0.24 \\
\hline 20 & MOL012266 & Rivularin & 22 & 37.94 & -0.13 & 0.37 \\
\hline 21 & MOL000507 & Psi-Baptigenin & 22 & 70.12 & -0.27 & 0.31 \\
\hline 22 & MOL001803 & Sinensetin & 21 & 50.56 & 0.04 & 0.45 \\
\hline 23 & MOL000552 & 5,2'-Dihydroxy-6,7,8-trimethoxyflavone & 21 & 31.71 & 0 & 0.35 \\
\hline 24 & MOL002927 & Skullcapflavone II & 21 & 69.51 & -0.07 & 0.44 \\
\hline 25 & MOL011078 & $3^{\prime}, 7$-dihydroxy-4' $4^{\prime}$-methoxy-isoflavone & 21 & 50.70 & -0.09 & 0.24 \\
\hline 26 & MOL003758 & Iristectorigenin (9CI) & 21 & 71.55 & -0.16 & 0.34 \\
\hline 27 & MOL003656 & Lupiwighteone & 21 & 51.64 & -0.23 & 0.37 \\
\hline 28 & MOL012101 & Mosloflavone & 19 & 34.04 & 0.29 & 0.26 \\
\hline 29 & MOL002563 & Galangin & 19 & 45.55 & -0.09 & 0.21 \\
\hline 30 & MOL004883 & Licoisoflavone & 19 & 41.61 & -0.27 & 0.42 \\
\hline 31 & MOL005012 & Licoagroisoflavone & 18 & 57.28 & 0.09 & 0.49 \\
\hline 32 & MOL002915 & Salvigenin & 18 & 49.07 & -0.03 & 0.33 \\
\hline 33 & MOL004848 & Licochalcone G & 17 & 49.25 & -0.04 & 0.32 \\
\hline 34 & MOL002917 & 5,2',6'-Trihydroxy-7,8-dimethoxyflavone & 17 & 45.05 & -0.11 & 0.33 \\
\hline 35 & MOL004884 & Licoisoflavone B & 17 & 38.93 & -0.18 & 0.55 \\
\hline 36 & MOL004564 & Kaempferid & 17 & 73.41 & -0.21 & 0.27 \\
\hline 37 & MOL005321 & Frutinone A & 16 & 65.90 & 0.46 & 0.34 \\
\hline 38 & MOL013279 & $5,7,4^{\prime}$-Trimethylapigenin & 16 & 39.83 & 0.12 & 0.30 \\
\hline 39 & MOL002235 & Eupatin & 16 & 50.80 & -0.26 & 0.41 \\
\hline 40 & MOL012108 & Negletein & 15 & 41.16 & 0.13 & 0.23 \\
\hline 41 & MOL008127 & Ermanin & 15 & 58.95 & 0.07 & 0.30 \\
\hline 42 & MOL005573 & Genkwanin & 14 & 37.13 & -0.24 & 0.24 \\
\hline 43 & MOL005849 & Didymin & 13 & 38.55 & -0.07 & 0.24 \\
\hline 44 & MOL000239 & Jaranol & 13 & 50.83 & -0.22 & 0.29 \\
\hline 45 & MOL004598 & 3,5,6,7-tetramethoxy-2-(3,4,5-trimethoxyphenyl)chromone & 12 & 31.97 & 0.08 & 0.59 \\
\hline 46 & MOL005842 & Pectolinarigenin & 12 & 41.17 & -0.09 & 0.30 \\
\hline 47 & MOL000525 & Norwogonin & 12 & 39.40 & -0.17 & 0.21 \\
\hline 48 & MOL004114 & $3,2^{\prime}, 4^{\prime}, 6^{\prime}$-Tetrahydroxy-4, $3^{\prime}$-dimethoxy chalcone & 11 & 52.69 & -0.15 & 0.28 \\
\hline 49 & MOL002341 & Hesperetin & 9 & 70.31 & -0.25 & 0.27 \\
\hline 50 & MOL006331 & $4^{\prime}, 5$-Dihydroxyflavone & 8 & 48.55 & -0.03 & 0.19 \\
\hline 51 & MOL002398 & Karanjin & 5 & 69.56 & 0.62 & 0.34 \\
\hline 52 & MOL000242 & 7-O-Methyleriodictyol & 5 & 56.56 & -0.21 & 0.27 \\
\hline 53 & MOL002913 & Dihydrobaicalin_qt & 4 & 40.04 & 0.18 & 0.21 \\
\hline 54 & MOL002908 & $5,8,2^{\prime}$-Trihydroxy-7-methoxyflavone & NA & 37.01 & -0.07 & 0.27 \\
\hline 55 & MOL002719 & 6-Hydroxynaringenin & NA & 33.23 & -0.27 & 0.24 \\
\hline
\end{tabular}

NA: not available.

edges were embodied with the average node degree 34.2. These results were imported into Cytoscape software (version 3.8.2) for further analysis. The network is shown in
Figure 3. The node color reflected the number of interacted nodes, and the more nodes to one node linked with, the deeper colored it became, as shown in Figure 4. 
TABle 3: Active flavonoids in the treatment of glioma reported in PubMed in recent five years.

\begin{tabular}{|c|c|c|c|c|}
\hline No. & Molecule ID & Molecule name & Related targets & Annotation \\
\hline 1 & MOL000098 & Quercetin & 154 & [14-19] \\
\hline 2 & MOL006821 & Epigallocatechin-3-gallate & 140 & {$[20,21]$} \\
\hline 3 & MOL001789 & Isoliquiritigenin & 124 & {$[22,23]$} \\
\hline 4 & MOL000481 & Genistein & 97 & {$[24]$} \\
\hline 5 & MOL000008 & Apigenin & 80 & [25-29] \\
\hline 6 & MOL000422 & Kaempferol & 63 & {$[30]$} \\
\hline 7 & MOL000006 & Luteolin & 57 & {$[31-35]$} \\
\hline 8 & MOL013179 & Fisetin & 46 & {$[36]$} \\
\hline 9 & MOL002008 & Myricetin & 38 & {$[37,38]$} \\
\hline 10 & MOL005734 & Eupatilin & 31 & [39] \\
\hline 11 & MOL005814 & Tangeretin & 29 & {$[40,41]$} \\
\hline 12 & MOL000417 & Calycosin & 22 & {$[42]$} \\
\hline 13 & MOL002560 & Chrysin & 19 & {$[43,44]$} \\
\hline 14 & MOL002083 & Tricin & 18 & {$[45]$} \\
\hline 15 & MOL009297 & Jaceosidin & 16 & {$[46]$} \\
\hline 16 & MOL005811 & Hepta-3 & 14 & [47] \\
\hline 17 & MOL000492 & $(+)$-Catechin & 11 & {$[2]$} \\
\hline 18 & MOL002881 & Diosmetin & 10 & [3] \\
\hline 19 & MOL005093 & Diosmin & 10 & [48] \\
\hline 20 & MOL005190 & Eriodictyol & 9 & [49] \\
\hline 21 & MOL013374 & Ampelopsin & 7 & [50] \\
\hline 22 & MOL004925 & Vitexin & 6 & {$[51,52]$} \\
\hline 23 & MOL002931 & Scutellarin & 6 & [53] \\
\hline 24 & MOL005812 & Naringin & 5 & {$[54,55]$} \\
\hline 25 & MOL007450 & Silybin & 2 & {$[43,56,57]$} \\
\hline 26 & MOL001790 & Linarin & 1 & {$[58,59]$} \\
\hline 27 & MOL004425 & Icariin & 1 & {$[60]$} \\
\hline 28 & MOL002037 & Amentoflavone & NA & {$[61-64]$} \\
\hline 29 & MOL007285 & Procyanidol C1 & NA & {$[65]$} \\
\hline
\end{tabular}

NA: not available.

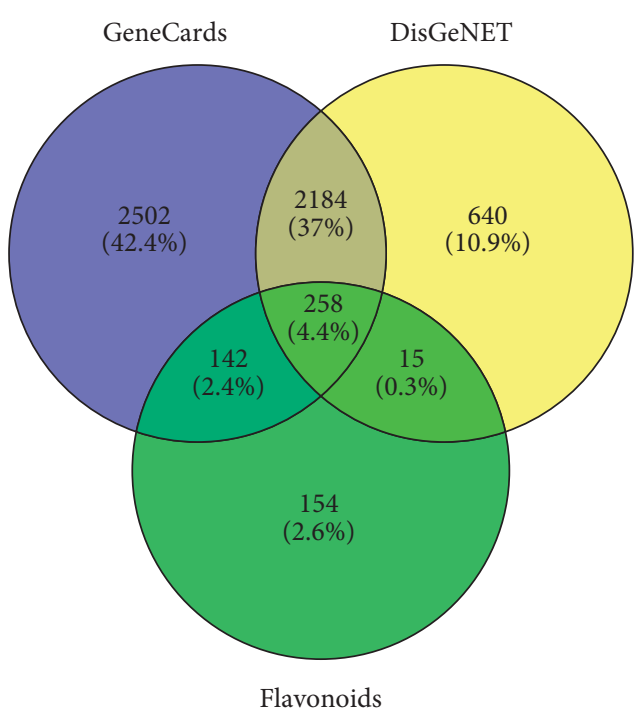

FIGURE 2: Overlapping target genes between glioma and flavonoids.

The potential targets were AKT1, EGFR, VEGFA, MAPK3, CASP3, SRC, HRAS, TNF, MAPK1, CCND1, ESR1, HSP90AA1, and MTOR as their degrees were above 100 (Table 4). Node degrees were counted by Cytoscape. The greater a node degree is, the more important biological functions the node has in the PPI network (Table 5).
3.4. GO and KEGG Pathway Enrichment Analyses. GO terms were enriched by the Metascape platform. The results showed that BP terms enriched in glioma-flavonoids overlapping targets mainly included peptidyl-tyrosine phosphorylation and modification, response to oxidative stress and oxygen levels. The top five enriched CC terms were membrane raft, membrane microdomain, membrane region, neuronal cell body, and transferase complex, transferring phosphorus-containing groups. MF terms displayed the intersection genes that were mainly enriched in protein tyrosine kinase activity, protein serine/threonine kinase activity, transmembrane receptor protein kinase activity, transmembrane receptor protein tyrosine kinase activity, and phosphatase binding (Figure 5).

KEGG pathway enrichment analysis of the 258 intersection gene targets was carried out by Metascape. The main pathways among these genes included PI3K-Akt, Ras, HIF-1, and Neurotrophin signaling pathways (Figure 6).

3.5. Molecular Docking. The result of the KEGG pathway enrichment analysis indicated that the PI3K-Akt signaling pathway was the main pathway through which flavonoids affected the glioma. Among the potential targets, AKT1, EGFR, MAPK1, MAPK3, CCND1, MTOR, VEGFA, HRAS, and HSP90AA1 were enriched in the PI3K-Akt signaling pathway. We selected seven potential active molecules, 


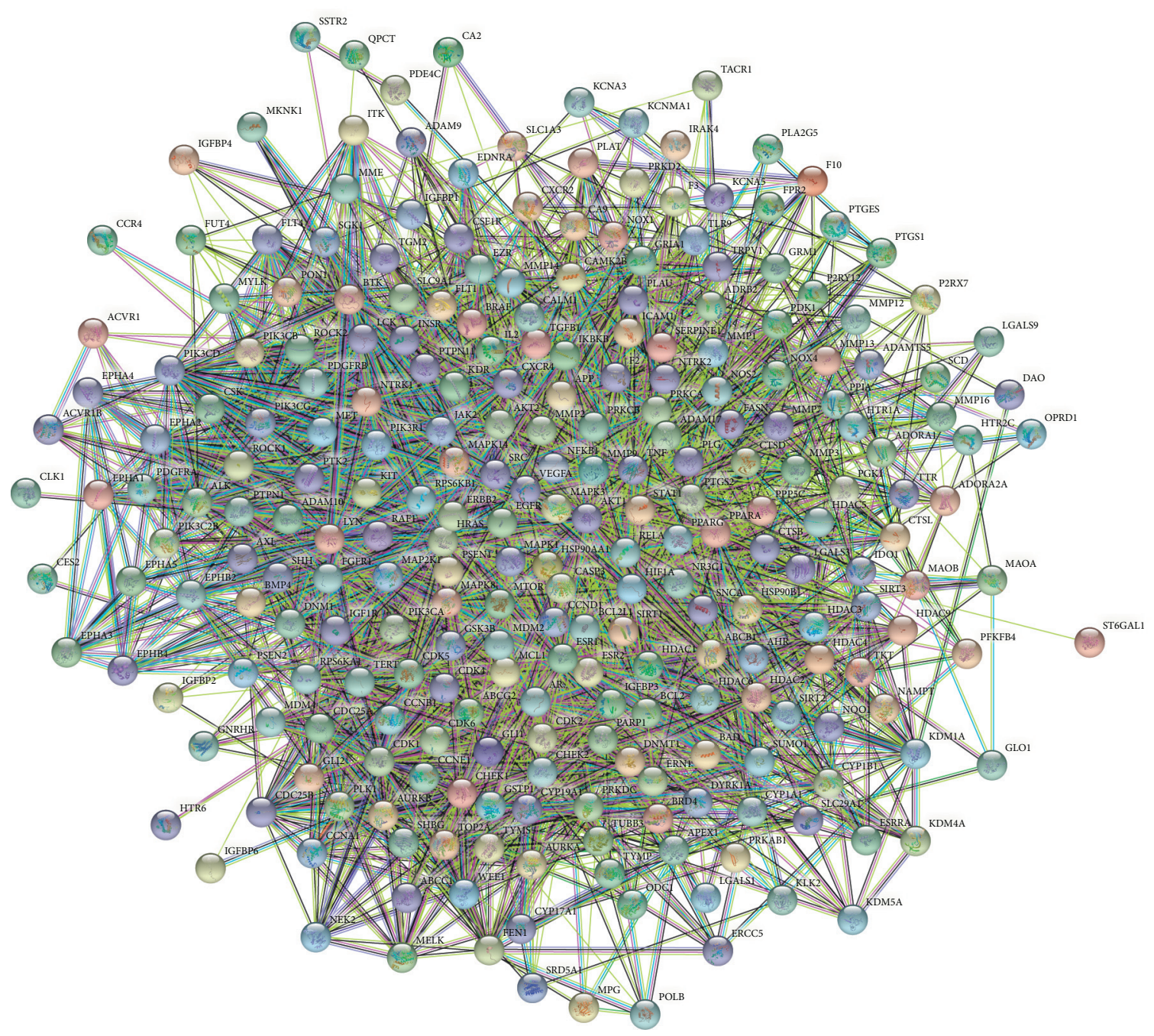

FIGURE 3: PPI network of potential targets generated by STRING (258 nodes, 4407 edges).

including quercetin, epigallocatechin-3-gallate, isoliquiritigenin, genistein, apigenin, kaempferol, and luteolin, to dock with nine target proteins, and chose temozolomide as the control. Lower binding energy indicates a stabler conformation. We used the quantitative value of fitness to evaluate the binding level. Fitness is the total energy of a predicted pose in the binding site. The empirical scoring function of iGEMDOCK is estimated as follows: Fitness $=\mathrm{vdW}+$ Hbond + Elec. The vdW term is van der Waal energy; Hbond and Elect terms are hydrogen bonding energy and electrostatic energy, respectively [66]. The results were visually displayed with a heatmap (Figure 7 ). It was interesting to note that epigallocatechin-3-gallate (EGCG) had a good bonding ability to most target proteins enriched in the PI3K-Akt signaling pathway, while the opposite pattern was observed for isoliquiritigenin. Experimental studies show that quercetin could induce autophagy and apoptosis in human neuroglioma cells through the PI3K-Akt signaling pathway [67]. AKT1, MTOR, CCND1, and EGFR are closely associated with autophagy and apoptosis in glioma [68-70]. Our findings obviously showed that EGCG had a better docking score to these proteins than quercetin.
For further research, quercetin and EGCG were selected in this study to dock with MAPK1 and HSP90AA1 target proteins individually due to their relatively lower energy value in the molecular docking. The results of SwissDock revealed that the estimated Gibbs free energies $(\Delta G)$ of best binding modes of EGCG with two targeting proteins were $-9.27 \mathrm{kcal} / \mathrm{mol}$ and $-8.53 \mathrm{kcal} / \mathrm{mol}$, respectively, while the binding energies of quercetin with two targeting proteins were $-8.23 \mathrm{kcal} / \mathrm{mol}$ and $-7.95 \mathrm{kcal} / \mathrm{mol}$, separately. In addition, EGCG had one backbone hydrogen bond (HB) interacting with Glu33 of MAPK1, and the distance was $2.031 \AA$. One backbone HB bounded with Gly97 of HSP90AA1 with a distance of $2.117 \AA$. The results displayed by UCSF Chimera software were shown in Figure 8.

\section{Discussion}

In recent years, flavonoids are widely used for antiglioma treatment. The mechanisms of flavonoids are very complex because they have multiple potential targets and active components. Network pharmacology together with bioinformatics has superiority in the systematic elucidation of 


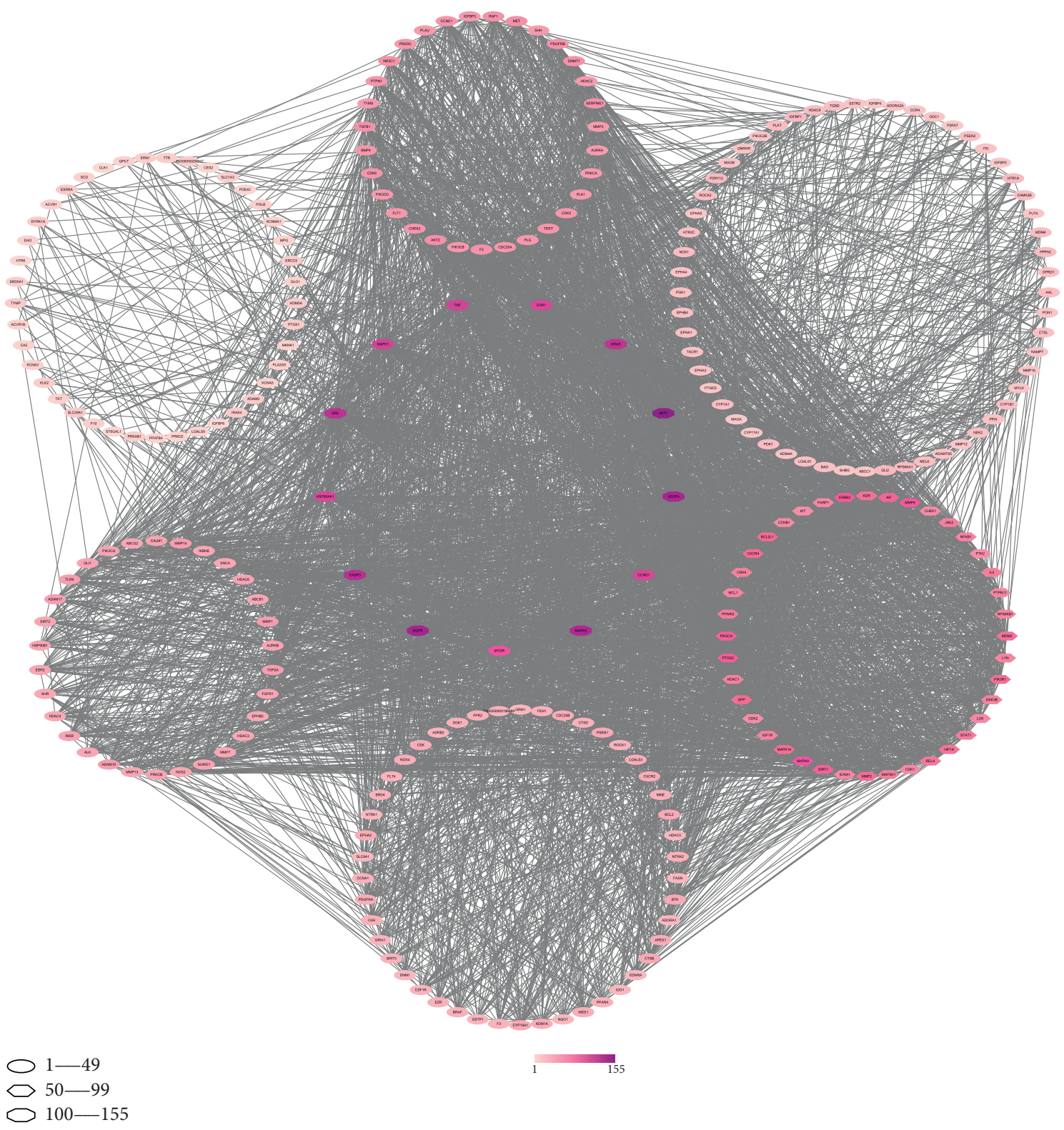

FIGURE 4: PPI network of common targets of flavonoids treating glioma. The node color was in proportion to the number of interacted nodes, and the more nodes the node linked, the deeper color it showed.

TABle 4: Potential active targets of flavonoids.

\begin{tabular}{|c|c|c|c|c|}
\hline No. & Uniprot ID & Target name & Protein name & Degree \\
\hline 1 & P31749 & AKT1 & RAC-alpha serine/threonine-protein kinase & 155 \\
\hline 2 & P00533 & EGFR & Epidermal growth factor receptor & 139 \\
\hline 3 & P15692 & VEGFA & Vascular endothelial growth factor A & 136 \\
\hline 4 & P27361 & MAPK3 & Mitogen-activated protein kinase 3 & 134 \\
\hline 5 & P42574 & CASP3 & Caspase-3 & 128 \\
\hline 6 & P12931 & SRC & Protooncogene tyrosine-protein kinase Src & 126 \\
\hline 7 & P01112 & HRAS & GTPase HRas & 124 \\
\hline 8 & P01375 & TNF & Tumor necrosis factor & 115 \\
\hline 9 & P28482 & MAPK1 & Mitogen-activated protein kinase 1 & 115 \\
\hline 10 & P24385 & CCND1 & G1/S-specific cyclin D1 & 112 \\
\hline 11 & P03372 & ESR1 & Estrogen receptor & 112 \\
\hline 12 & P07900 & HSP90AA1 & Heat Shock Protein HSP 90-alpha & 111 \\
\hline 13 & P42345 & MTOR & Serine/threonine-protein kinase mTOR & 104 \\
\hline
\end{tabular}


TABLE 5: Docking score of targets with seven active ingredients ( $\mathrm{kcal} / \mathrm{mol})$.

\begin{tabular}{lccccccccc}
\hline Target name & PDB ID & Apigenin & EGCG & Genistein & Isoliquiritigenin & Kaempferol & Luteolin & Quercetin & Temozolomide \\
\hline AKT1 & 6S9W & -87.88 & -104.05 & -103.38 & -84.38 & -91.79 & -94.33 & -90.26 & -101.62 \\
EGFR & 7AEM & -96.74 & -114.52 & -106.53 & -93.64 & -100.48 & -98.17 & -95.28 & -117.90 \\
MAPK1 & 6RFP & -97.85 & -143.55 & -98.34 & -94.73 & -96.12 & -95.77 & -106.79 & -113.58 \\
MAPK3 & 6GES & -97.51 & -102.29 & -101.62 & -94.60 & -93.60 & -99.52 & -98.23 & -107.29 \\
CCND1 & 3AY5 & -83.32 & -96.44 & -87.54 & -83.87 & -80.69 & -86.00 & -87.49 & -90.09 \\
MTOR & 7JWE & -99.64 & -107.48 & -90.22 & -82.14 & -89.54 & -100.05 & -96.34 & -97.78 \\
VEGFA & 6D3O & -84.52 & -98.62 & -85.47 & -80.56 & -85.32 & -87.91 & -86.79 & -86.84 \\
HRAS & 4XVR & -98.48 & -97.18 & -98.35 & -77.91 & -96.60 & -99.33 & -96.30 & -104.24 \\
HSP90AA1 & 4BQG & -90.22 & -123.19 & -98.78 & -92.72 & -100.26 & -94.73 & -84.84 & -98.88 \\
\hline
\end{tabular}

the mechanism of TCM at the molecular level and representation of interactions between active compounds, potential targets, and various pathways.

In our study, potential targets of active components analysis revealed that quercetin, EGCG, isoliquiritigenin, genistein, apigenin, kaempferol, and luteolin interacted with multiple targets in the network. These findings showed that they may play important roles in the treatment of glioma. As reported, these seven active flavonoid compounds have distinct ways of treating glioma. Quercetin, a flavonol, had the most potential targets in this study. It plays antiglioma effects by inducing cell apoptosis [17], inhibiting proliferation and migration [14], and modulating the inflammatory process [71]. Moreover, quercetin could affect human glioma cells through the PI3K-Akt signaling pathway [72]. EGCG is a polyphenol flavonoid, which is generally distributed in green tea and has shown great properties in cancer prevention due to its safety, low cost, and excellent bioavailability [73]. EGCG in high doses ( $>40 \mu \mathrm{mol} / \mathrm{L}$ ) could suppress cancer cells by inducing apoptosis and by inhibiting autophagic processes [74] and regulate apoptosis-related and autophagy-related proteins (caspase3, caspase 9, Bax, LC3B II, and Beclin) [75]. MTOR is a key regulator of autophagy, EGCG may enhance the phosphorylation of eNOS and mTOR via the activation of the PI3K-Akt pathway [76]. Furthermore, it has the effects of antiglioma through inhibiting proliferation and decreasing invasion of glioma cells [77]. Isoliquiritigenin, isolated from licorice, has been found to be a potent stimulator of cell differentiation and has potential application for treating human brain glioma by inhibiting proliferation and blocking angiogenic through Notch1 and Akt signaling pathway, respectively [22, 23]. Genistein, an isoflavone in legumes and some herbal medicines, suppresses the expression of matrix metalloproteinase 2 (MMP-2) and vascular endothelial growth factor (VEGF) to serve antigiloma role [78]. Genistein sensitizes glioblastoma cells to carbon ions through inhibiting DNA-PKcs phosphorylation and subsequently repressing the nonhomologous end-joining and delaying the homologous recombination repair pathways [24]. Apigenin, a flavone, has been shown to take part in restoring the immune system and weakening the selfrenewal and invasiveness capacity of glioblastoma stem-like cells (GSCs) $[25,29]$. It was reported to inhibit the expression of STAT3, AKT, and MAPK in the GSCs [26]. Kaempferol has also been demonstrated to possess good antiglioma effects by inducing reactive oxygen species (ROS) and subsequently leads to autophagy and cell death $[30,79]$. Luteolin is a flavone and has an inhibitory effect on downstream signal molecules activated by EGFR, particularly the Akt and MAPK signal pathways $[33,80]$. It induces a lethal endoplasmic reticulum stress response and mitochondrial dysfunction in glioblastoma cells by increasing intracellular ROS levels [31].

Immune factors have been considered as a significant factor contributing to the development and progression of glioma [81]. In the PPI network, most potential targets were closely related to immunity, including AKT1, TNF, EGFR, VEGFA, MAPK1, MAPK3, CASP3, SRC, HRAS, CCND1, ESR1, HSP90AA1, and MTOR [82-87]. And these proteins were regarded as core proteins in our study and might play important roles in the therapeutic effect of flavonoids on glioma. Recent studies have shown that luteolin decreased the expression of immune-related genes including MMP9, MAPK1, HSP90AA1, CASP3, ALB, EGFR, SRC, HRAS, and ESR1. And among these genes, MMP9, MAPK1, HSP90AA1, EGFR, SRC, and HRAS are confirmed in vivo at the protein and mRNA levels [88].

To further indicate the potential mechanism of flavonoids in treating glioma, KEGG analysis discovered that PI3K-Akt was the main signaling pathway. It is a classic signal transduction pathway involved in cell proliferation, apoptosis, migration, invasion, and angiogenesis in glioma and plays an important role in the occurrence and development of glioma [89]. The result of molecular docking showed that EGCG had good bonding with MAPK1 and HSP90AA1 in the PI3K-Akt signaling pathway. Relevant studies confirmed that EGCG induces apoptosis, inhibits proliferation, and decreases invasion of glioma cells via the MAPK pathway in vivo [77]. Kim et al. also found that EGCG induced the expression of MAPK1 in glioma cells [90]. Heat Shock Protein 90 can promote oncogenesis since it interacts and supports numerous proteins and is essential for malignant transformation and progression. However, the HSP90AA1 gene is not altered in a major of tumors according to the Cancer Genome Atlas (TCGA) [91]. To evaluate its role in the treatment of glioma, downregulation of HSP90AA1-IT1 (HSP90AA1 intronic transcript (1) was done, which could significantly suppress cell viability, proliferation, EMT, invasion, and migration of glioma [92]. Thus, there might be a correlation between HSP90AA1 and glioma; however, there is no report about EGCG curing glioma via targeting HSP90AA1.

Although there is an abundance of information and the analysis process is complex, some useful and credible 


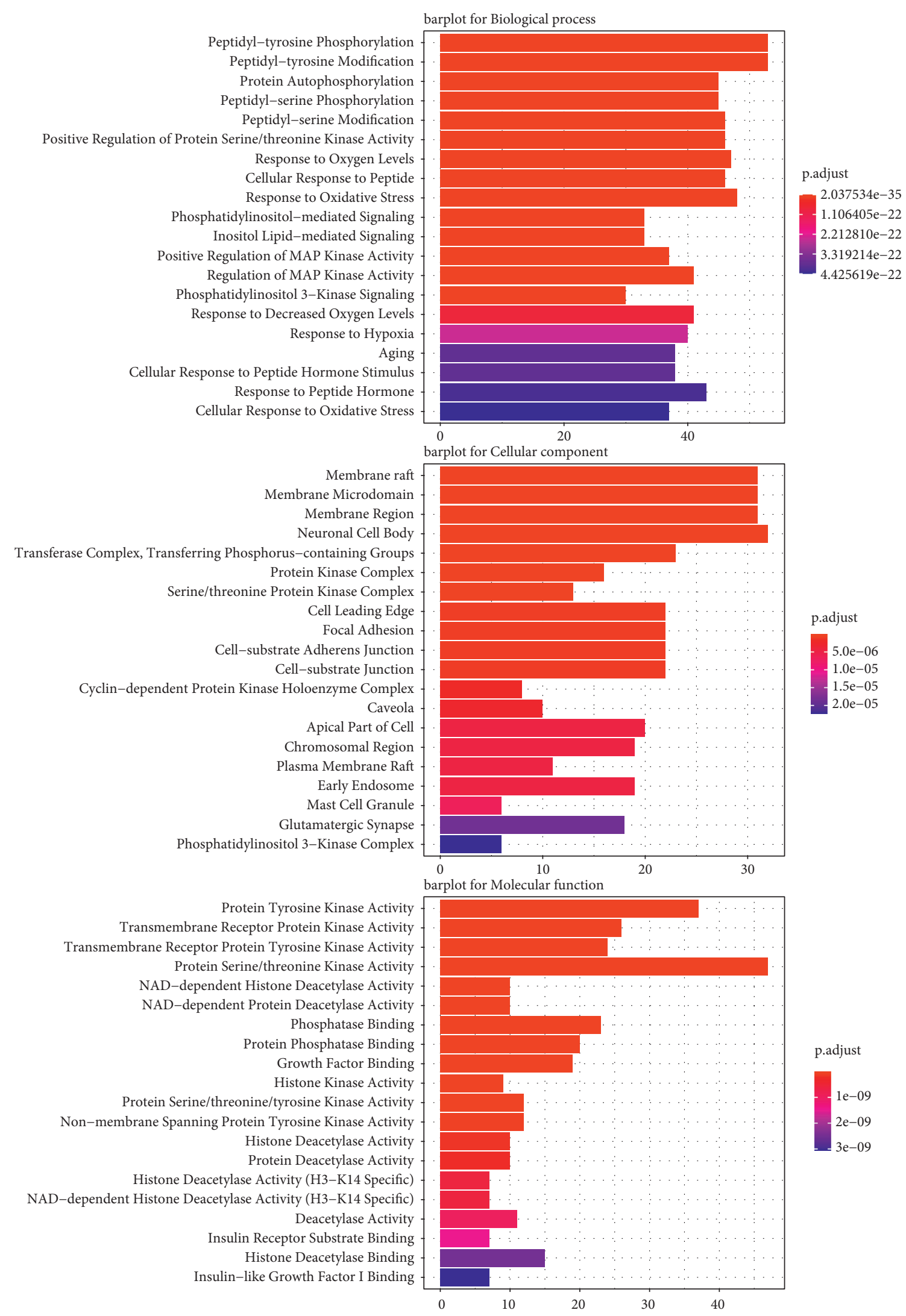

FIGURE 5: GO enrichment analysis of biological process (BP) terms, cellular component (CC) terms, and molecular function (MF) terms. The color of the bar is displayed in a gradient from red to blue according to the ascending order of the $P$ adjust, while the length of the bar is arranged according to the ascending order of the number of gene counts. 


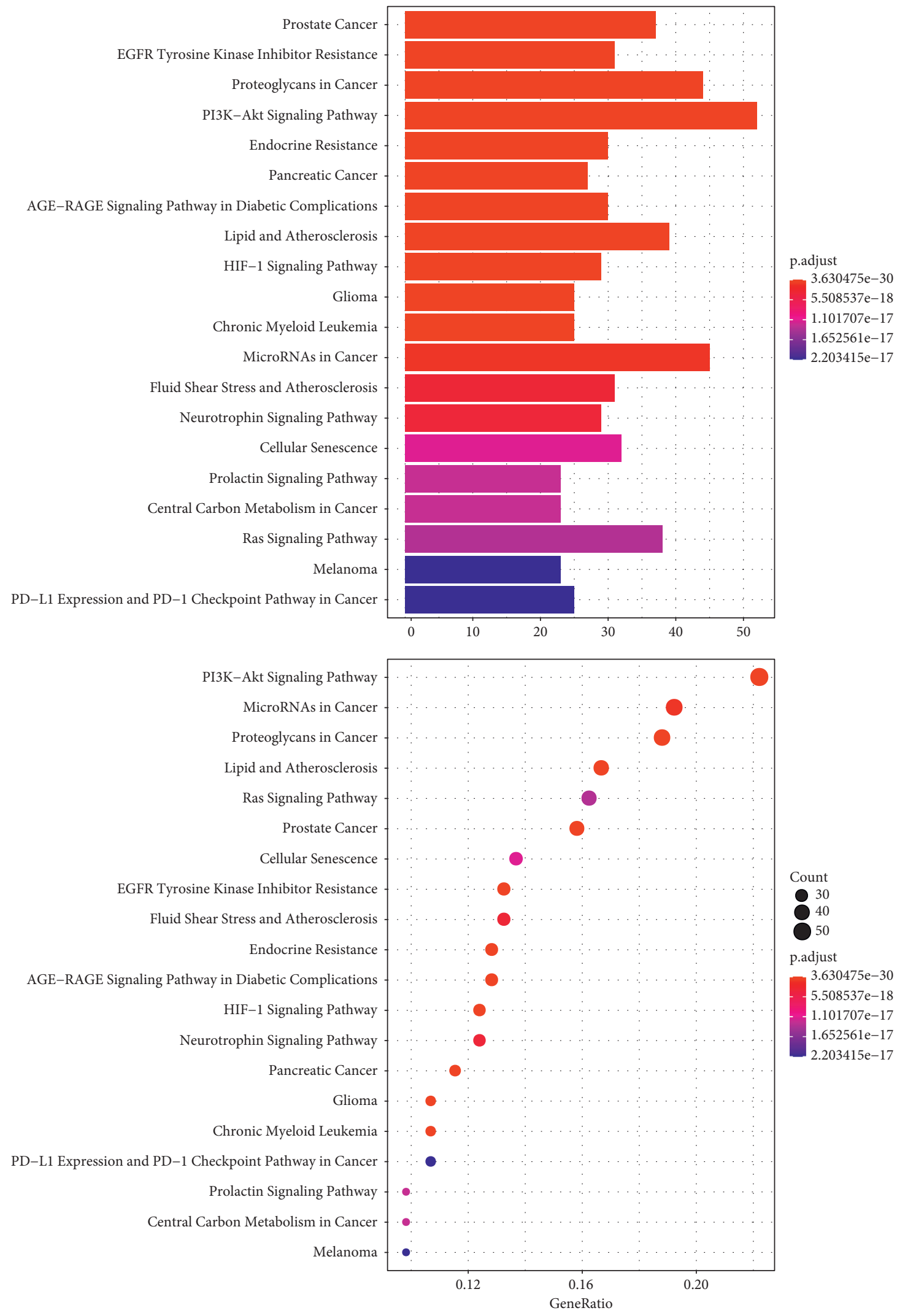

FIGURE 6: KEGG pathway analysis of potential targets of flavonoids on glioma-related proteins visualized by the clusterProfiler. The color of the bar is displayed in a gradient from red to blue according to the $P$ adjust, while the sizes of dots are arranged according to the ascending order of the number of gene counts. 


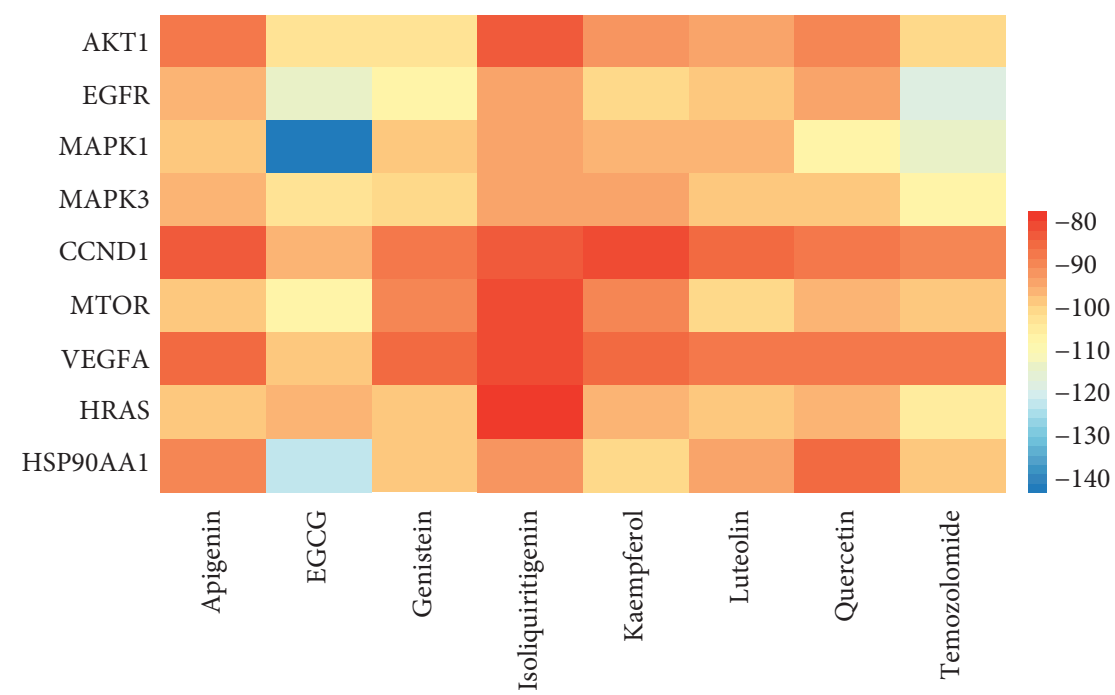

Figure 7: Heatmap of molecular docking. Temozolomide was taken as the control. The red color represents a high docking score, and blue represents a low docking score. The lowest value indicates the most stable conformation.

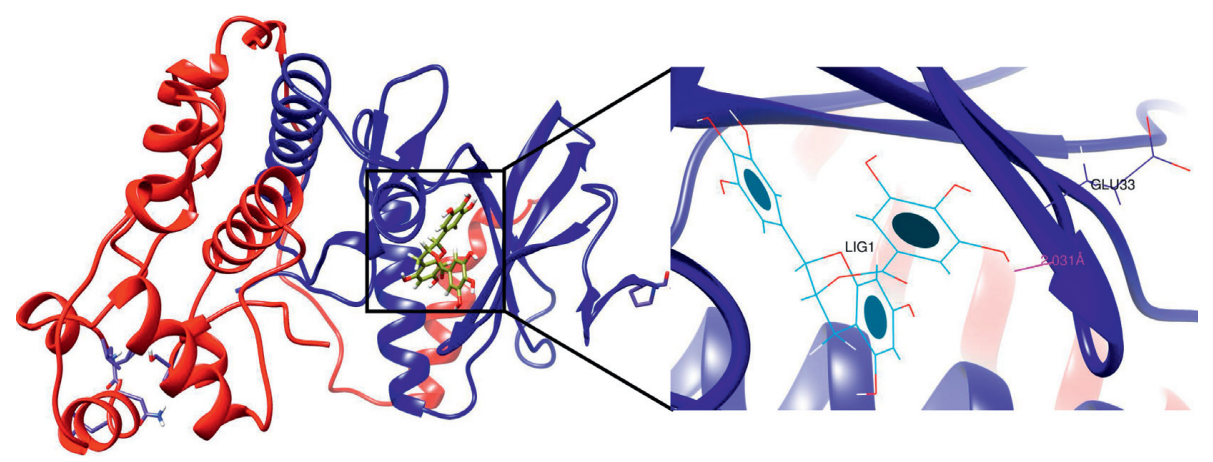

(a)

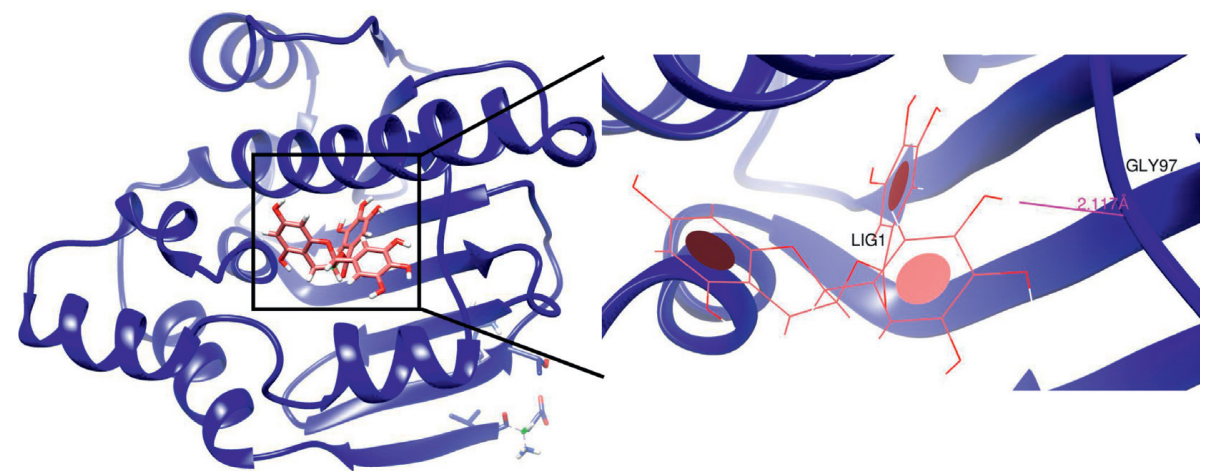

(b)

FIGURE 8: The binding modes of EGCG with MAPK1 (a) and HSP90AA1 (b). Left panel: this area showed the best combination pocket of EGCG with MAPK1 and HSP90AA1 proteins. EGCG and relevant residues were presented in stick representation. Right panel: this region showed the hydrogen bonding with Glu33 in MAPK1 protein and Gly97 in HSP90AA1 protein. The distance of hydrogen bond interaction was colored in pink, and the structure of EGCG was shown in circle and disk shape.

conclusions have been drawn. Due to limitations of compounds screening and accuracy of target prediction, the results obtained in this study are general, and in vitro and in vivo experiments are needed for verification. In short, our study portrayed the ground view of flavonoids in the treatment of glioma. 


\section{Conclusions}

This study elaborated the mechanisms of active flavonoids on antiglioma using network pharmacology and molecular docking by constructing a compound-target-pathway network. Active components have particular advantages in curing glioma by targeting MAPK1, MAPK3, EGFR, MTOR, AKT1, VEGFA, CCND1, HSP90AA1, and HRAS. In addition, EGCG can target HSP90AA1 and MAPK1 via the PI3K-Akt signaling pathway. These findings offered a research foundation for further investigation of flavonoids on antiglioma.

$\begin{array}{ll}\text { Abbreviations } \\ \text { BBB: } & \text { Blood-brain barrier } \\ \text { BP: } & \text { Biological process } \\ \text { CC: } & \text { Cell component } \\ \text { DL: } & \text { Drug-likeness } \\ \text { EGCG: } & \text { Epigallocatechin-3-gallate } \\ \text { EMT: } & \text { Epithelial-mesenchymal transition } \\ \text { eNOS: } & \text { Endothelial nitric oxide synthase } \\ \text { GO: } & \text { Gene Ontology } \\ \text { GSCs: } & \text { Glioblastoma stem-like cells } \\ \text { HB: } & \text { Hydrogen bond } \\ \text { HIF-1: } & \text { Hypoxia-inducible factor-1 } \\ \text { KEGG: } & \text { Kyoto Encyclopedia of Genes and Genomes } \\ \text { MF: } & \text { Molecular function } \\ \text { MMP-2: } & \text { Matrix metalloproteinase } 2 \\ \text { OB: } & \text { Oral bioavailability } \\ \text { PDB: } & \text { Protein data bank } \\ \text { PI3K- } & \text { Phosphatidylinositol 3-kinase-protein kinase B } \\ \text { Akt: } & \\ \text { PPI: } & \text { Protein-protein interaction } \\ \text { ROS: } & \text { Reactive oxygen species } \\ \text { SDF: } & \text { Structure-data file } \\ \text { STAT3: } & \text { Signal transducer and activator of transcription } 3 \\ \text { TCM: } & \text { Traditional Chinese medicine } \\ \text { TCMSP: } & \text { Traditional Chinese medicine database and } \\ & \text { analysis platform } \\ \text { AG: } & \text { Gibbs free energy. } \\ & \end{array}$

\section{Data Availability}

The data used to support the findings of this study are available from the corresponding author upon request.

\section{Conflicts of Interest}

The authors declare no conflicts of interest.

\section{Authors' Contributions}

Hong Xiao and Hongyi Liu designed the study. Taiping Li and Yong Xiao participated in the study design and wrote the article. Zhen Wang conducted data analysis. All authors read and approved the final manuscript. Taiping Li and Yong Xiao contributed equally to this work.

\section{Acknowledgments}

This study was supported by the Medical Research Foundation of Jiangsu Health Commission (H2019059), Science and Technology Development Foundation of Nanjing Medical University (NMUB2018095), Medical Science and Technology Development Foundation of Nanjing (YKK19088), and Postgraduate Research \& Practice Innovation Program of Jiangsu Province (SJCX19-0331 and KYCX20-1419). In addition, the authors are very grateful to Dr. Wang (Lijun Wang) for her careful revision of our manuscript.

\section{References}

[1] F. Zeng, G. Li, X. Liu et al., "Plasminogen activator urokinase receptor implies immunosuppressive features and acts as an unfavorable prognostic biomarker in glioma," The Oncologist, vol. 26, no. 8, 2021.

[2] Z. Sheng, "Anticancer effects of catechin flavonoid in human glioma cells are mediated via autophagy induction, cell cycle arrest, inhibition of cell migration and invasion and targeting MAPK/ERK signalling pathway," Official Journal of the Balkan Union of Oncology, vol. 25, no. 2, pp. 1084-1090, 2020.

[3] Y. Yan, X. Liu, J. Gao, and Y. Wu, "Dracocephalum peregrinum inhibition of TGF- $\beta$ signaling in gliomas by the flavonoid diosmetin isolated from L," Molecules, vol. 25, no. 1, 2020.

[4] A. Atiq and I. Parhar, "Anti-neoplastic potential of flavonoids and polysaccharide phytochemicals in glioblastoma," Molecules, vol. 2521 pages, 2020.

[5] T.-t. Luo, Y. Lu, S.-k. Yan, X. Xiao, X.-l. Rong, and J. Guo, "Network pharmacology in research of Chinese medicine formula: methodology, application and prospective," Chinese Journal of Integrative Medicine, vol. 26, no. 1, pp. 72-80, 2020.

[6] X. Ma, M. Yu, C. Hao, and W. Yang, "Shikonin induces tumor apoptosis in glioma cells via endoplasmic reticulum stress, and Bax/Bak mediated mitochondrial outer membrane permeability," Journal of Ethnopharmacology, vol. 263, Article ID 113059, 2020.

[7] L. Zhang, L. Han, and X. Wang, "Exploring the mechanisms underlying the therapeutic effect of Salvia miltiorrhiza on diabetic nephropathy using network pharmacology and molecular docking," Bioscience Reports, vol. 41, no. 6, 2021.

[8] Y. Du, Z. Yang, and Q. Sun, "Engineered microglia potentiate the action of drugs against glioma through extracellular vesicles and tunneling nanotubes," Advanced Healthcare Materials, vol. 10, no. 6, p. e2002200, 2021.

[9] L. Hu, Y. Chen, T. Chen, D. Huang, S. Li, and S. Cui, "A systematic study of mechanism of sargentodoxa cuneata and patrinia scabiosifolia against pelvic inflammatory disease with dampness-heat stasis syndrome via network pharmacology approach," Frontiers in Pharmacology, vol. 11, Article ID 582520, 2020.

[10] S. Zhang, X. Wang, F. Cheng et al., "Network pharmacologybased approach to revealing biological mechanisms of qingkailing injection against ischemic stroke: focusing on blood-brain barrier," Evidence-Based Complementary and Alternative Medicine, vol. 2020, Article ID 2914579, 16 pages, 2020.

[11] The Gene Ontology Consortium, "The gene ontology resource: 20 years and still going strong," Nucleic Acids Research, vol. 47, no. D1, pp. D330-D338, 2019. 
[12] J. Du, Z. Yuan, Z. Ma, J. Song, X. Xie, and Y. Chen, "KEGGPATH: Kyoto encyclopedia of genes and genomes-based pathway analysis using a path analysis model," Molecular BioSystems, vol. 10, no. 9, pp. 2441-2447, 2014.

[13] G. Yu, L.-G. Wang, Y. Han, and Q.-Y. He, "clusterProfiler: an $\mathrm{R}$ package for comparing biological themes among gene clusters," OMICS: A Journal of Integrative Biology, vol. 16, no. 5, pp. 284-287, 2012.

[14] Y. Liu, Z.-G. Tang, Y. Lin et al., "Effects of quercetin on proliferation and migration of human glioblastoma U251 cells," Biomedicine \& Pharmacotherapy, vol. 92, pp. 33-38, 2017.

[15] M. Kruszewski, M. Kusaczuk, J. Kotyńska et al., “The effect of quercetin on the electrical properties of model lipid membranes and human glioblastoma cells," Bioelectrochemistry, vol. 124, pp. 133-141, 2018.

[16] F. Damiano, L. Giannotti, G. V. Gnoni, L. Siculella, and A. Gnoni, "Quercetin inhibition of SREBPs and ChREBP expression results in reduced cholesterol and fatty acid synthesis in C6 glioma cells," The International Journal of Biochemistry \& Cell Biology, vol. 117, Article ID 105618, 2019.

[17] M. A. Taylor, F. Khathayer, and S. K. Ray, "Quercetin and sodium butyrate synergistically increase apoptosis in rat C6 and human T98G glioblastoma cells through inhibition of autophagy," Neurochemical Research, vol. 44, no. 7, pp. 1715-1725, 2019.

[18] M. Ersoz, A. Erdemir, S. Derman, T. Arasoglu, and B. Mansuroglu, "Quercetin-loaded nanoparticles enhance cytotoxicity and antioxidant activity on C6 glioma cells," Pharmaceutical Development and Technology, vol. 25, no. 6, pp. 757-766, 2020.

[19] E. Jang, I. Y. Kim, H. Kim et al., "Quercetin and chloroquine synergistically kill glioma cells by inducing organelle stress and disrupting $\mathrm{Ca} 2+$ homeostasis," Biochemical Pharmacology, vol. 178, Article ID 114098, 2020.

[20] C. T. Le, W. P. J. Leenders, R. J. Molenaar, and C. J. F. van Noorden, "Effects of the green tea polyphenol epigallocatechin-3-gallate on glioma: a critical evaluation of the literature," Nutrition and Cancer, vol. 70, no. 3, pp. 317-333, 2018.

[21] I. Udroiu, J. Marinaccio, and A. Sgura, "Epigallocatechin-3gallate induces telomere shortening and clastogenic damage in glioblastoma cells," Environmental and Molecular Mutagenesis, vol. 60, no. 8, pp. 683-692, 2019.

[22] Y. Lin, H. Sun, Y. Dang, and Z. Li, "Isoliquiritigenin inhibits the proliferation and induces the differentiation of human glioma stem cells," Oncology Reports, vol. 39, no. 2, pp. 687-694, 2018.

[23] C. Wang, Y. Chen, Y. Wang et al., "Inhibition of COX-2, mPGES-1 and CYP4A by isoliquiritigenin blocks the angiogenic Akt signaling in glioma through ceRNA effect of miR-194-5p and lncRNA NEAT1," Journal of Experimental \& Clinical Cancer Research, vol. 38, no. 1, p. 371, 2019.

[24] X. Liu, P. Li, R. Hirayama et al., "Genistein sensitizes glioblastoma cells to carbon ions via inhibiting DNA-PKcs phosphorylation and subsequently repressing NHEJ and delaying HR repair pathways," Radiotherapy \& Oncology, vol. 129, no. 1, pp. 84-94, 2018.

[25] P. L. C. Coelho, M. N. Oliveira, A. B. da Silva et al., "The flavonoid apigenin from Croton betulaster Mull inhibits proliferation, induces differentiation and regulates the inflammatory profile of glioma cells," Anti-Cancer Drugs, vol. 27 , no. 10 , pp. 960-969, 2016.

[26] B. Kim, N. Jung, S. Lee, J. K. Sohng, and H. J. Jung, “Apigenin inhibits cancer stem cell-like phenotypes in human glioblastoma cells via suppression of c-met signaling," Phytotherapy Research, vol. 30, no. 11, pp. 1833-1840, 2016.

[27] X.-J. Chen, M.-Y. Wu, D.-H. Li, and J. You, "Apigenin inhibits glioma cell growth through promoting microRNA-16 and suppression of BCL-2 and nuclear factor- $\kappa$ B/MMP-9," Molecular Medicine Reports, vol. 14, no. 3, pp. 2352-2358, 2016.

[28] T. A. Stump, B. N. Santee, L. P. Williams et al., "The antiproliferative and apoptotic effects of apigenin on glioblastoma cells," Journal of Pharmacy and Pharmacology, vol. 69, no. 7, pp. 907-916, 2017.

[29] P. L. C. Coelho, J. A. O. Amparo, A. B. Silva et al., “Apigenin from Croton betulaster Müll restores the immune profile of microglia against glioma cells," Phytotherapy Research, vol. 33, no. 12, pp. 3191-3202, 2019.

[30] M. Colombo, F. Figueiró, A. de Fraga Dias, H. F. Teixeira, A. M. O. Battastini, and L. S. Koester, "Kaempferol-loaded mucoadhesive nanoemulsion for intranasal administration reduces glioma growth in vitro," International Journal of Pharmaceutics, vol. 543, no. 1-2, pp. 214-223, 2018.

[31] Q. Wang, H. Wang, Y. Jia, H. Pan, and H. Ding, "Luteolin induces apoptosis by ROS/ER stress and mitochondrial dysfunction in gliomablastoma," Cancer Chemotherapy and Pharmacology, vol. 79, no. 5, pp. 1031-1041, 2017.

[32] D. El Gueder, M. Maatouk, Z. Kalboussi et al., "Heat processing effect of luteolin on anti-metastasis activity of human glioblastoma cells U87," Environmental Science and Pollution Research, vol. 25, no. 36, pp. 36545-36554, 2018.

[33] D. M. Anson, R. M. Wilcox, E. D. Huseman et al., "Luteolin decreases epidermal growth factor receptor-mediated cell proliferation and induces apoptosis in glioblastoma cell lines," Basic and Clinical Pharmacology and Toxicology, vol. 123, no. 6, pp. 678-686, 2018.

[34] C. Yi, G. Li, D. N. Ivanov et al., "Luteolin inhibits Musashi1 binding to RNA and disrupts cancer phenotypes in glioblastoma cells," RNA Biology, vol. 15, no. 11, pp. 1420-1432, 2018.

[35] C. Wu, Q. Xu, X. Chen, and J. Liu, "Delivery luteolin with folacin-modified nanoparticle for glioma therapy," International Journal of Nanomedicine, vol. 14, pp. 7515-7531, 2019.

[36] F. Pak and P. Oztopcu-Vatan, "Fisetin effects on cell proliferation and apoptosis in glioma cells," Zeitschrift für Naturforschung C, vol. 74, no. 11-12, pp. 295-302, 2019.

[37] H.-F. Zhao, G. Wang, C.-P. Wu et al., "A multi-targeted natural flavonoid myricetin suppresses lamellipodia and focal adhesions formation and impedes glioblastoma cell invasiveness and abnormal motility," CNS \& Neurological Disorders-Drug Targets, vol. 17, no. 7, pp. 557-567, 2018.

[38] G. Wang, J.-J. Wang, Y.-Z. Wang, S. Feng, G. Jing, and X.-L. Fu, "Myricetin nanoliposomes induced SIRT3-mediated glycolytic metabolism leading to glioblastoma cell death," Artificial Cells, Nanomedicine, and Biotechnology, vol. 46, no. 3, pp. S180-S191, 2018.

[39] X. Fei, J. Wang, C. Chen et al., "Eupatilin inhibits glioma proliferation, migration, and invasion by arresting cell cycle at G1/S phase and disrupting the cytoskeletal structure," Cancer Management and Research, vol. 11, pp. 4781-4796, 2019.

[40] L.-L. Ma, D.-w. Wang, X.-D. Yu, and Y.-L. Zhou, “Tangeretin induces cell cycle arrest and apoptosis through upregulation of PTEN expression in glioma cells," Biomedicine \& Pharmacotherapy, vol. 81, pp. 491-496, 2016.

[41] Y. P. Cheng, S. Li, W. L. Chuang et al., "Blockade of STAT3 signaling contributes to anticancer effect of 5-acetyloxy$6,7,8,4^{\prime}$-tetra-methoxyflavone, a tangeretin derivative, on human glioblastoma multiforme cells," International Journal of Molecular Sciences, vol. 20, no. 13, 2019. 
[42] X. Nie, Y. Zhou, and X. Li, "Calycosin down-regulates c-Met to suppress development of glioblastomas," Journal of Biosciences, vol. 44, no. 4, 2019.

[43] M. Gülden, D. Appel, M. Syska, S. Uecker, F. Wages, and H. Seibert, "Chrysin and silibinin sensitize human glioblastoma cells for arsenic trioxide," Food and Chemical Toxicology: An International Journal Published for the British Industrial Biological Research Association, vol. 105, pp. 486497, 2017.

[44] J. Wang, H. Wang, K. Sun et al., "Chrysin suppresses proliferation, migration, and invasion in glioblastoma cell lines via mediating the ERK/Nrf2 signaling pathway," Drug Design, Development and Therapy, vol. 12, pp. 721-733, 2018.

[45] D.-J. Chung, C.-J. Wang, C.-W. Yeh, and T.-H. Tseng, "Inhibition of the proliferation and invasion of C6 glioma cells by tricin via the upregulation of focal-adhesion-kinase-targeting MicroRNA-7," Journal of Agricultural and Food Chemistry, vol. 66, no. 26, pp. 6708-6716, 2018.

[46] S. M. Woo and T. K. Kwon, "Jaceosidin induces apoptosis through Bax activation and down-regulation of Mcl-1 and c-FLIP expression in human renal carcinoma Caki cells," Chemico-Biological Interactions, vol. 260, pp. 168-175, 2016.

[47] A. Sawamoto, S. Okuyama, M. Nakajima, and Y. Furukawa, "Citrus flavonoid 3,5,6,7,8,3',4'-heptamethoxyflavone induces BDNF via CAMP/ERK/CREB signaling and reduces phosphodiesterase activity in C6 cells," Pharmacological Reports, vol. 71, no. 4, pp. 653-658, 2019.

[48] J. M. Soares, B. M. Faria, L. M. Ascari et al., "Diosmin induces caspase-dependent apoptosis in human glioblastoma cells," Anais da Academia Brasileira de Ciencias, vol. 91, no. 4, 2019.

[49] W. Li, Q. Du, X. Li et al., "Eriodictyol inhibits proliferation, metastasis and induces apoptosis of glioma cells via PI3K/Akt/ NF- $\kappa$ B signaling pathway," Frontiers in Pharmacology, vol. 11, p. 114, 2020.

[50] Z. Guo, H. Guozhang, H. Wang, Z. Li, and N. Liu, "Ampelopsin inhibits human glioma through inducing apoptosis and autophagy dependent on ROS generation and JNK pathway," Biomedicine \& Pharmacotherapy, vol. 116, Article ID 108524, 2019.

[51] G. Zhang, D. Li, and H. Chen, "Vitexin induces G2/M-phase arrest and apoptosis via Akt/mTOR signaling pathway in human glioblastoma cells," Molecular Medicine Reports, vol. 17, no. 3, pp. 4599-4604, 2018.

[52] T. Xie, J.-R. Wang, C.-G. Dai, X.-A. Fu, J. Dong, and Q. Huang, "Vitexin, an inhibitor of hypoxia-inducible factor$1 \alpha$, enhances the radiotherapy sensitization of hyperbaric oxygen on glioma," Clinical and Translational Oncology, vol. 22, no. 7, pp. 1086-1093, 2020.

[53] X. He, L. Xiong, and Q. Xia, "CHNO and its combination with scutellarin suppress the proliferation and induce the apoptosis of human glioma cells via upregulation of fas-associated factor 1 expression," BioMed Research International, vol. 2019, Article ID 6821219, 20 pages, 2019.

[54] J. Li, Y. Dong, G. Hao et al., "Naringin suppresses the development of glioblastoma by inhibiting FAK activity," Journal of Drug Targeting, vol. 25, no. 1, pp. 41-48, 2017.

[55] S. Aroui, H. Fetoui, and A. Kenani, "Natural dietary compound naringin inhibits glioblastoma cancer neoangiogenesis," BMC Pharmacology and Toxicology, vol. 21, no. 1, p. $46,2020$.

[56] Z. Bai, V. Tay, and S. Guo, "Silibinin induced human glioblastoma cell apoptosis concomitant with autophagy through simultaneous inhibition of mTOR and YAP," BioMed
Research International, vol. 2018, Article ID 6165192, 10 pages, 2018.

[57] C. Wang, C. He, S. Lu et al., "Autophagy activated by silibinin contributes to glioma cell death via induction of oxidative stress-mediated BNIP3-dependent nuclear translocation of AIF," Cell Death \& Disease, vol. 11, no. 8, p. 630, 2020.

[58] Z.-G. Zhen, S.-H. Ren, H.-M. Ji et al., "Linarin suppresses glioma through inhibition of NF- $\kappa \mathrm{B} / \mathrm{p} 65$ and up-regulating p53 expression in vitro and in vivo," Biomedicine \& Pharmacotherapy, vol. 95, pp. 363-374, 2017.

[59] Z.-f. Xu, X.-k. Sun, Y. Lan, C. Han, Y.-d. Zhang, and G. Chen, "Linarin sensitizes tumor necrosis factor-related apoptosis (TRAIL)-induced ligand-triggered apoptosis in human glioma cells and in xenograft nude mice," Biomedicine \& Pharmacotherapy, vol. 95, pp. 1607-1618, 2017.

[60] Y. Yang and G. Li, "Icariin inhibits proliferation, migration, and invasion of medulloblastoma DAOY cells by regulation of SPARC," Phytotherapy Research, vol. 34, no. 3, pp. 591-600, 2020.

[61] T. H. Yen, C. L. Hsieh, T. T. Liu et al., “Amentoflavone induces apoptosis and inhibits NF-KB-modulated anti-apoptotic signaling in glioblastoma cells," In Vivo (Athens, Greece), vol. 32, no. 2, pp. 279-285, 2018.

[62] W. Zhaohui, N. Yingli, L. Hongli et al., "Amentoflavone induces apoptosis and suppresses glycolysis in glioma cells by targeting miR-124-3p," Neuroscience Letters, vol. 686, pp. 1-9, 2018.

[63] F. Hsu, I. Chiang, and Y. Kuo, "Amentoflavone effectively blocked the tumor progression of glioblastoma via suppression of ERK/NF- B signaling pathway," The American journal of Chinese medicine, vol. 47, no. 4, pp. 913-931, 2019.

[64] Y. Chen, N. Li, H. Wang et al., "Amentoflavone suppresses cell proliferation and induces cell death through triggering autophagy-dependent ferroptosis in human glioma," Life Sciences, vol. 247, Article ID 117425, 2020.

[65] D.-L. ZhuGe, L.-F. Wang, R. Chen et al., "Cross-linked nanoparticles of silk fibroin with proanthocyanidins as a promising vehicle of indocyanine green for photo-thermal therapy of glioma," Artificial cells, nanomedicine, and biotechnology, vol. 47, no. 1, pp. 4293-4304, 2019.

[66] K. Hsu, Y. Chen, S. Lin, and J. Yang, "iGEMDOCK: a graphical environment of enhancing GEMDOCK using pharmacological interactions and post-screening analysis," BMC Bioinformatics, vol. S33, 2011.

[67] M. Lou, L.-n. Zhang, P.-g. Ji et al., "Quercetin nanoparticles induced autophagy and apoptosis through AKT/ERK/Caspase-3 signaling pathway in human neuroglioma cells: in vitro and in vivo," Biomedicine \& Pharmacotherapy, vol. 84, pp. 1-9, 2016.

[68] K. Fujiwara, E. Iwado, G. Mills, R. Sawaya, S. Kondo, and Y. Kondo, "Akt inhibitor shows anticancer and radiosensitizing effects in malignant glioma cells by inducing autophagy," International Journal of Oncology, vol. 31, no. 4, pp. 753-760, 2007.

[69] Y. Cheng and P. Xie, "Ganoderic acid A holds promising cytotoxicity on human glioblastoma mediated by incurring apoptosis and autophagy and inactivating PI3K/AKT signaling pathway," Journal of Biochemical and Molecular Toxicology, vol. 33, no. 11, Article ID e22392, 2019.

[70] Y. Liu, L. Yang, F. Liao, W. Wang, and Z.-F. Wang, "MiR$450 a-5 p$ strengthens the drug sensitivity of gefitinib in glioma chemotherapy via regulating autophagy by targeting EGFR," Oncogene, vol. 39, no. 39, pp. 6190-6202, 2020. 
[71] A. B. da Silva, P. L. Cerqueira Coelho, M. das Neves Oliveira et al., "The flavonoid rutin and its aglycone quercetin modulate the microglia inflammatory profile improving antiglioma activity," Brain, Behavior, and Immunity, vol. 85, pp. 170-185, 2020.

[72] H.-C. Pan, Q. Jiang, Y. Yu, J.-P. Mei, Y.-K. Cui, and W.-J. Zhao, "Quercetin promotes cell apoptosis and inhibits the expression of MMP-9 and fibronectin via the AKT and ERK signalling pathways in human glioma cells," Neurochemistry International, vol. 80, pp. 60-71, 2015.

[73] B. N. Singh, S. Shankar, and R. K. Srivastava, "Green tea catechin, epigallocatechin-3-gallate (EGCG): mechanisms, perspectives and clinical applications," Biochemical Pharmacology, vol. 82, no. 12, pp. 1807-1821, 2011.

[74] L. Chen, H.-L. Ye, G. Zhang et al., "Autophagy inhibition contributes to the synergistic interaction between EGCG and doxorubicin to kill the hepatoma Hep3B cells," PLoS One, vol. 9, no. 1, Article ID e85771, 2014.

[75] Z. Yin, J. Li, L. Kang et al., "Epigallocatechin-3-gallate induces autophagy-related apoptosis associated with LC3B II and beclin expression of bladder cancer cells," Journal of Food Biochemistry, vol. 45, no. 6, Article ID e13758, 2021.

[76] F. Xuan and J. Jian, "Epigallocatechin gallate exerts protective effects against myocardial ischemia/reperfusion injury through the PI3K/Akt pathway-mediated inhibition of apoptosis and the restoration of the autophagic flux," International Journal of Molecular Medicine, vol. 38, no. 1, pp. 328-336, 2016.

[77] H. Li, Z. Li, Y.-M. Xu et al., "Epigallocatechin-3-gallate induces apoptosis, inhibits proliferation and decreases invasion of glioma cell," Neuroscience Bulletin, vol. 30, no. 1, pp. 67-73, 2014.

[78] Y. Yazdani, M. R. Sharifi Rad, M. Taghipour, N. Chenari, A. Ghaderi, and M. Razmkhah, "Genistein suppression of matrix metalloproteinase 2 (MMP-2) and vascular endothelial growth factor (VEGF) expression in mesenchymal stem cell like cells isolated from high and low grade gliomas," Asian Pacific Journal of Cancer Prevention, vol. 17, no. 12, pp. 5303-5307, 2016.

[79] S. Chen, J. Ma, L. Yang et al., "Anti-glioblastoma activity of kaempferol via programmed cell death induction: involvement of autophagy and pyroptosis," Frontiers in Bioengineering and Biotechnology, vol. 8, Article ID 614419, 2020.

[80] Y. You, R. Wang, N. Shao, F. Zhi, and Y. Yang, "Luteolin suppresses tumor proliferation through inducing apoptosis and autophagy via MAPK activation in glioma," OncoTargets and Therapy, vol. 12, pp. 2383-2396, 2019.

[81] Y. Feng, J. Wang, D. Tan, P. Cheng, and A. Wu, "Relationship between circulating inflammatory factors and glioma risk and prognosis: a meta-analysis," Cancer Medicine, vol. 8, no. 17, pp. 7454-7468, 2019.

[82] F. Tao, J. Zhu, L. Duan et al., "Anti-inflammatory effects of doxepin hydrochloride against LPS-induced C6-glioma cell inflammatory reaction by PI3K-mediated Akt signaling," Journal of Biochemical and Molecular Toxicology, vol. 34, no. 2, Article ID e22424, 2020.

[83] E. Madan, B. Dikshit, S. H. Gowda et al., "FAT1 is a novel upstream regulator of HIF $1 \alpha$ and invasion of high grade glioma," International Journal of Cancer, vol. 139, no. 11, pp. 2570-2582, 2016.

[84] T. Li, J. Zhao, J. Ge et al., "Particulate matter facilitates C6 glioma cells activation and the release of inflammatory factors through MAPK and JAK2/STAT3 pathways," Neurochemical Research, vol. 41, no. 8, pp. 1969-1981, 2016.
[85] D. Xu, T. Kong, Z. Shao et al., "Orexin-A alleviates astrocytic apoptosis and inflammation via inhibiting OX1R-mediated NF- $\kappa \mathrm{B}$ and MAPK signaling pathways in cerebral ischemia/ reperfusion injury," Biochimica et Biophysica Acta-Molecular Basis of Disease, vol. 1867, no. 11, Article ID 166230, 2021.

[86] K. Fatih, K. Sedat, and H. Ceyhan, "Concanavalin A induces apoptosis in a dose-dependent manner by modulating thiol/ disulfide homeostasis in C6 glioblastoma cells," Journal of Biochemical and Molecular Toxicology, vol. 35, no. 5, 2021.

[87] A. González-Morales, A. Zabaleta, and M. García-Moure, "Oncolytic adenovirus delta-24-RGD induces a widespread glioma proteotype remodeling during autophagy," Journal of Proteomics, vol. 194, pp. 168-178, 2019.

[88] S. Gao, Z. Sha, J. Zhou et al., "BYSL contributes to tumor growth by cooperating with the mTORC2 complex in gliomas," Cancer Biology and Medicine, vol. 18, no. 1, pp. 88-104, 2021.

[89] X.-F. Huang, J.-L. Zhang, D.-P. Huang et al., "A network pharmacology strategy to investigate the anti-inflammatory mechanism of luteolin combined with in vitro transcriptomics and proteomics," International Immunopharmacology, vol. 86, Article ID 106727, 2020.

[90] S.-J. Kim, H.-J. Jeong, K.-M. Lee et al., "Epigallocatechin-3gallate suppresses NF- $\kappa \mathrm{B}$ activation and phosphorylation of p38 MAPK and JNK in human astrocytoma U373MG cells," The Journal of Nutritional Biochemistry, vol. 18, no. 9, pp. 587-596, 2007.

[91] A. D. Zuehlke, K. Beebe, L. Neckers, and T. Prince, "Regulation and function of the human HSP90AAl gene," Gene, vol. 570, no. 1, pp. 8-16, 2015.

[92] T. Gao, G. Gu, J. Tian et al., "LncRNA HSP90AA1-IT1 promotes gliomas by targeting miR-885-5p-CDK2 pathway," Oncotarget, vol. 8, no. 43, pp. 75284-75297, 2017. 